\title{
Research
}

\section{Cost effectiveness of case-finding strategies for primary prevention of cardiovascular disease:}

\author{
a modelling study
}

\begin{abstract}
Background

Policies of active case finding for cardiovascular disease (CVD) prevention in healthy adults are common, but economic evaluation has not investigated targeting such strategies at those who are most likely to benefit.

\section{Aim}

To assess the cost effectiveness of targeted case finding for CVD prevention.

\section{Design and setting}

Cost-effectiveness modelling in an English primary care population.

\section{Method}

A cohort of 10000 individuals aged $30-74$ years and without existing CVD or diabetes was sampled from The Health Improvement Network database, a large primary care database. A discrete-event simulation was used to model the process of inviting people for assessment, assessing cardiovascular risk, and initiation and persistence with drug treatment. Risk factors and drug cessation rates were obtained from primary care data. Published sources provided estimates of uptake of assessment, treatment initiation, and treatment effects. The researchers determined the lifetime costs and quality-adjusted life years (QALYS) with opportunistic case finding, and strategies prioritising and targeting patients by age or prior estimate of cardiovascular risk. This study reports on the optimum strategy if a $\mathrm{QALY}$ is valued at $€ 20000$.

\section{Results}

Compared with no case finding, inviting all adults aged $30-74$ years in a population of 10000 yields 30.32 QALYs at a total cost of $£ 705$ 732. The optimum strategy is to rank

\section{INTRODUCTION}

Patients can be identified for cardiovascular disease (CVD) prevention in primary care opportunistically or through active CVD risk factor assessment (case finding). Opportunistic identification means assessing CVD risk factors and considering whether patients are eligible for prevention when they consult for other reasons. Many healthcare systems in developed countries recommend that CVD risk factor assessment is routinely offered to all otherwise healthy middle-aged adults. This is recommended for men aged $\geq 40$ years and women aged $\geq 50$ years in Canadian and European guidelines; for most Australians aged $\geq 45$ years; and in the UK from age 40 years. ${ }^{1-4}$ The policy is being considered or implemented in middle-income countries such as Kuwait, Malaysia, and Lebanon. ${ }^{5-7}$

Scepticism remains about active case finding. The case for the policy rests on the evidence that antihypertensive treatments and statins are effective, and the assumption that sufficient eligible patients will be found and treated through risk factor assessment, and therefore sufficient CVD prevented to justify the policy of identification. Assessment has little value if assessed patients are unlikely to be treated. Clinical trials do not support widespread CVD risk factor assessment ${ }^{8-9}$ and recent evaluation of the UK Health Check programmes found only modest short-term effects on CVD risk
\end{abstract} patients by prior risk estimate and invite $8 \%$ of those who are assessed as being at highest risk lthose at $\geq 12.76 \%$ predicted 10 -year CVD risk), yielding 17.53 QALYS at a cost of $€ 162280$ There is an $89.4 \%$ probability that the optimum strategy is to invite $<35 \%$ of patients for assessment.

\section{Conclusion}

Across all age ranges, targeted case finding using a prior estimate of CVD risk is more efficient than universal case finding in healthy adults.

\section{Keywords}

cardiovascular diseases; cost-effectiveness; primary care; risk stratification; screening.
C Crossan, MSc, research fellow, Health Economics Research Group, Brunel University London, Uxbridge. J Lord, PhD, director, Southampton Health Technology Assessments Centre, University of Southampton, Southampton. R Ryan, PhD, research fellow, Primary Care Clinical Sciences; T Marshall, PhD, professor of public health and primary care, School of Health and Population Sciences, University of Birmingham, Birmingham. L Nherera, MSc, health economics manager, Smith \& Nephew, Hull. factors. ${ }^{10}$ In the UK, economic modelling concluded that case finding across all those aged $40-74$ years was highly cost effective compared with opportunistic assessment. ${ }^{11}$ However, as targeting selected patients for CVD prevention by age or by an estimated risk score was not modelled, the strategies compared were case finding in all or in none of the population - as such, the incremental health gains and costs of inviting selected patients were not investigated. This does not reflect the real policy options for case finding because risk factor assessment could be offered to any selected proportion of the population, be that $1 \%$ or $99 \%$.

In many countries lincluding the Netherlands, Denmark, Norway, Sweden, Finland, Iceland, Spain, Portugal, France, New Zealand, and the UKI primary care providers have electronic patient records (EPRs). Providers can use information from the EPR (age, sex, CVD risk factors) to prioritise and select patients for CVD prevention. This study compared the cost effectiveness of three strategies for identifying and treating those at risk of CVD in English primary care:

- a base case of opportunistic assessment; - active invitation of an increasing proportion of the population for CVD risk assessment prioritised by their age (oldest first); and

\section{Address for correspondence}

Tom Marshall, School of Health and Population Sciences, University of Birmingham, Edgbaston, Birmingham, B15 2TT, UK.

E-mail: T.P.Marshallabham.ac.uk

Submitted: 13 July 2016; Editor's response: 30 July 2016; final acceptance: 9 August 2016 (CBritish Journal of General Practice

This is the full-length article (published online 8 Nov 2016) of an abridged version published in print. Cite this version as: Br J Gen Pract 2016; DOI: 10.3399/bjgp16X687973 


\section{How this fits in}

Health check programmes for prevention of cardiovascular disease are common in the UK and other countries. Proponents claim the strategy identifies and treats patients eligible for antihypertensives and statins. However, a strategy of targeted, rather than universal, health checks has not been evaluated. This study revealed that most of the benefits of case finding can be achieved at a fraction of the cost by targeting patients at highest risk of developing CVD.

- active invitation based on a prior estimate of CVD risk that was calculated using age, sex, and other existing information from the individual's clinical records (highest risk first).

\section{METHOD}

An individual-level discrete-event simulation was used to model the processes of:

- case finding;

- assessment;

- initiation of preventive treatment (statins and antihypertensives); and

- continuation with treatment for a cohort of patients (Figure 1).

Discrete-event simulation is a method of modelling a sequence of events. Patient attributes and events that happen earlier in the sequence (such as treatment) affect the attributes and probability of events that happen later in the sequence (such as developing diabetes or cardiovascular risk). It allows resource costs and health outcomes to be modelled.

Methods advocated by the National Institute for Health and Care Excellence (NICE) ${ }^{12}$ were used: an NHS cost perspective was adopted, and lifetime impacts on the incidence of CVD, diabetes, and treatmentrelated adverse events were estimated.

Findings were expressed as costs (UK E sterling at 2014/2015 prices) and qualityadjusted life years (QALYS), both discounted at an annual rate of $3.5 \%$ (the discount rate recommended by NICE).

Input parameters and data sources are available from the authors on request. The model was implemented in Stata Iversion 13) and probabilistic sensitivity analysis was used to explore assumptions and uncertainties.

\section{Study population}

A cohort of 10000 individuals was sampled (with replacement) from all 1067654 patients in The Health Improvement
Network (THIN) database on 31 December 2013, who were:

- aged 30-74 years;

- without a clinical code indicating CVD or diabetes; and

- not currently receiving a prescription for blood pressure or lipid-lowering drugs.

Patients with chronic kidney disease were included as it was not possible to determine whether they had previously declined antihypertensives or statins; however, this group accounted for only $0.5 \%$ of the study population.

THIN is an anonymised database of medical records from 562 general practices, broadly representative of the UK population. For each individual, comprehensive baseline risk factor information including age, sex, ethnicity, comorbidities, and measured risk factors was extracted. Blood pressure and cholesterol measurements for each individual were calculated as an average of their most recent three measurements. If categorical risk factors - such as smoking status, comorbidities, and family history of premature coronary heart disease - were missing, this was assumed to indicate their absence.

Two estimates of 10-year CVD risk (prior risk and true risk) at model entry were calculated by the researchers for each individual using the $\mathrm{QRISK}^{\circledR} 2$ algorithm recommended by NICE. ${ }^{13}$ Prior risk was estimated using individuals' baseline risk factor information extracted from THIN and allowing QRISK2 to default to the population mean values for any missing risk factor information. These estimates, therefore, reflect the partial information available in real-life primary care records, and were used to select patients to invite for full CVD risk assessment under the targeted casefinding strategies.

True CVD risk at model entry was estimated by applying the QRISK2 algorithm to an imputed dataset, modelling the effect of complete risk factor information. Truncated regression (which avoids imputing values outside a plausible rangel was used to impute missing values for body mass index (BMI), blood pressure, cholesterol, and the Townsend deprivation index. The true CVD risk was used in the model to simulate the incidence of CVD events. A similar approach was used to estimate a true risk for type 2 diabetes. $^{14}$

\section{Case-finding strategies}

Two active case-finding strategies lone based on age, one based on prior risk) 


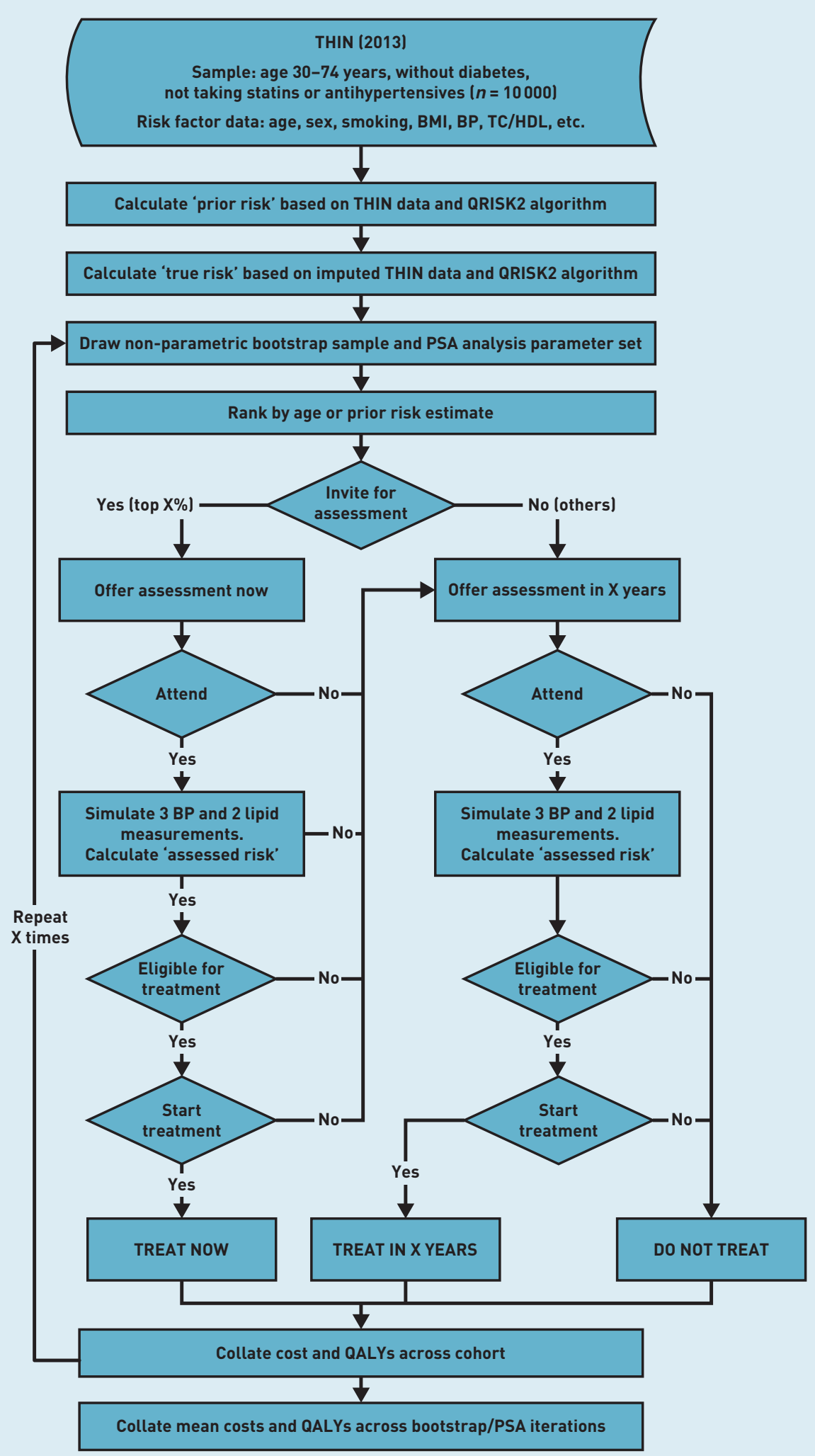

Figure 1. Discrete-event simulation flowchart.

$B M I=$ body mass index. $B P=$ blood pressure. $P S A=$ probabilistic sensitivity analysis. $Q A L Y=$ quality-adjusted life year. $T C / H D L=$ total cholesterol/high-density lipoprotein. $T H I N=$ The Health Improvement Network database. were modelled, each at varying levels of implementation, from inviting a few of the patients with the highest priority through to universal screening. These active strategies were compared with opportunistic risk assessment. It was assumed that opportunistic risk assessment would also continue alongside active case finding, meaning those not invited or who did not attend for active assessment could be identified opportunistically at a later date. Annual assessment rates, estimated from a previous study, were $61.9 \%$ for those actively invited and $4.3 \%$ for those invited opportunistically. ${ }^{15}$

Existing software (for example, EMIS or MSDi Clinical Manager) can generate lists of patients ranked either by age or prior risk. For active case-finding strategies, additional costs per person invited were estimated to account for administration time and the time GPs spent generating and checking the list of patients. Standard sources were used for staff costs. (For example, consultation costs come from the Personal Social Services Research Unit.) Further information is available from the authors on request.

\section{Clinical risk assessment}

Guidelines recommend that patients undergo CVD risk assessment before being prescribed a statin or antihypertensive drug. ${ }^{13,16}$ It was assumed that this included one consultation each with a healthcare assistant and a practice nurse, as well as recommended laboratory tests of lipid profile, liver function tests, glycosylated haemoglobin, and renal function. ${ }^{13}$

As measured CVD risk factors vary, risk factor assessment can misclassify patients as eligible or ineligible for treatments. The outcome of a CVD risk assessment was mimicked by simulating blood pressure and lipid measurements based on individuals lipid and blood pressure records lusing imputed values where necessaryl, plus random error terms to reflect intraindividual variation. The QRISK2 equation was then applied to the simulated lipid and blood pressure measurements, along with other risk factor information to obtain an estimated assessed risk.

\section{Treatment eligibility and initiation}

Treatment eligibility was based on assessed risk. Patients at $\geq 10 \% 10$-year CVD risk are eligible for statins. ${ }^{13}$ In sensitivity analyses, this was varied to range from $7.5 \%$ (to reflect guidance from the US) to $20.0 \% .{ }^{17}$ It was assumed that patients at $\geq 20 \% 10$-year CVD risk would be offered antihypertensives: one antihypertensive if their blood pressure was 


\section{Table 1. Sample characteristics ${ }^{\mathrm{a}, \mathrm{b}}$}

\begin{tabular}{|c|c|c|c|}
\hline Characteristic & Missing data, $n(\%)$ & Observed value & Imputed value \\
\hline Age & & $48(10.96)^{c}$ & \\
\hline Male, $n(\%)$ & & 525400 (49) & \\
\hline Ethnicity, $n(\%)$ & 499346 (46.8) & & \\
\hline White & & 304533 (28.5) & \\
\hline Indian & & 17401 (1.63) & \\
\hline Pakistani & & $7090(0.66)$ & \\
\hline Bangladeshi & & $1866(0.17)$ & \\
\hline Other Indian & & $8581(0.80)$ & \\
\hline Black Caribbean & & $4706(0.44)$ & \\
\hline Black African & & $12316(1.15)$ & \\
\hline Chinese & & 3933 (0.37) & \\
\hline Other ethnic group & & 207882 (19.5) & \\
\hline Smoking status, $n(\%)$ & 45091 (4) & & \\
\hline Non-smoker & & 604889 (57) & \\
\hline Ex-smoker & & $212865(20)$ & \\
\hline Current smoker & & 204809 (19) & \\
\hline Townsend index & $55370(5)$ & $-0.92(3.84)^{c}$ & $-0.91(3.85)^{c}$ \\
\hline Body mass index & $156686(15)$ & $26.37(5.19)^{c}$ & $26.37(5.19)^{c}$ \\
\hline Systolic blood pressure & 93989 (9) & $125.46(14.69)^{c}$ & $125.42(14.68)^{\mathrm{c}}$ \\
\hline Diastolic blood pressure & 94080 (9) & $77.01(9.44)^{c}$ & $77.01(9.43)^{c}$ \\
\hline Total cholesterol & $581011(54)$ & $5.29(0.98)^{c}$ & $5.17(0.98)^{c}$ \\
\hline HDL cholesterol & 632782 (59) & $1.47(0.44)^{c}$ & $1.45(0.43)^{c}$ \\
\hline Family history CVD, $n(\%)$ & & $5214(0.15 \%)$ & \\
\hline Family history diabetes, $n(\%)$ & & $9288(0.87 \%)$ & \\
\hline Atrial fibrillation, $n(\%)$ & & $1626(0.15 \%)$ & \\
\hline Rheumatoid arthritis, $n(\%)$ & & $7330(0.69 \%)$ & \\
\hline Chronic kidney disease, $n(\%)$ & & $5366(0.50 \%)$ & \\
\hline Taking corticosteroids, $n(\%)$ & & $115186(11 \%)$ & \\
\hline
\end{tabular}

a Population aged 30-74 years, without CVD, diabetes, or prescriptions for antihypertensives or statins in THIN database (total dataset for 2013: 1067 654). ${ }^{\circ}$ For diagnoses and other categorical variables, the absence of a clinical code is taken to mean the condition is absent. ${ }^{c}$ Mean (standard deviation). CVD = cardiovascular disease. $T H I N=$ The Health Improvement Network. events were modified by relative risks from published meta-analyses. Effects of statins were expressed per $1.00 \mathrm{mmol} / \mathrm{L}$ reduction in low-density lipoprotein cholesterol. ${ }^{18}$ Effects of antihypertensive medications were estimated from the reduction in systolic blood pressure (SBP) obtained with standard doses of one, two, or three antihypertensive drugs; ${ }^{19,20}$ the effects of these SBP reductions on cardiovascular events were then estimated. ${ }^{2}$

\section{Treatment cessation and adverse effects}

As the relative risk reductions for statins and antihypertensive drugs already incorporate diluted effects due to nonadherence by clinical trial participants, no additional modelling to account for imperfect medication adherence was undertaken. However, treatment cessation was modelled to reflect more realistic drop-out rates in routine practice. Rates of treatment cessation la gap in prescribing of $\geq 120$ days) for both statins and antihypertensives were estimated from a Kaplan-Meier survival analysis of UK primary care data. For statins and antihypertensives respectively, the mean probability of a patient continuing with treatment was:

- 0.861 (95\% confidence interval $[\mathrm{Cl}]=0.857$ to 0.866 ) for statins at 1 year;

- $0.688(95 \% \mathrm{Cl}=0.681$ to 0.694$)$ for statins at 5 years;

- $0.706(95 \% \mathrm{Cl}=0.696$ to 0.715$)$ for antihypertensives at 1 year; and

- $0.491(95 \% \mathrm{Cl}=0.479$ to 0.502$)$ for antihypertensives at 5 years.

Statins may be associated with an increased incidence of type 2 diabetes, muscle pain or myopathy, and abnormal liver function tests. ${ }^{22,23}$ The researchers took a conservative approach, including estimated increased risk of diabetes, myopathy, and liver dysfunction from a systematic review of observational studies..$^{23}$ Putative protective effects of statins on other chronic diseases, such as colorectal cancer or dementia, were excluded.

A range of adverse effects are associated with antihypertensive drugs, which may cause patients to stop taking the medication. ${ }^{24,25}$ It was assumed that, after permanently discontinuing treatment, patients would experience no significant cost or lasting health impact.

\section{Long-term health outcomes and costs}

A discrete-event approach was used to simulate the:

Individuals' baseline risks of cardiovascular 
Figure 2. Mean cost and QALY gain per 10000 population compared with no active case finding. $C V D=$ cardiovascular disease. $Q A L Y=$ qualityadjusted life year.

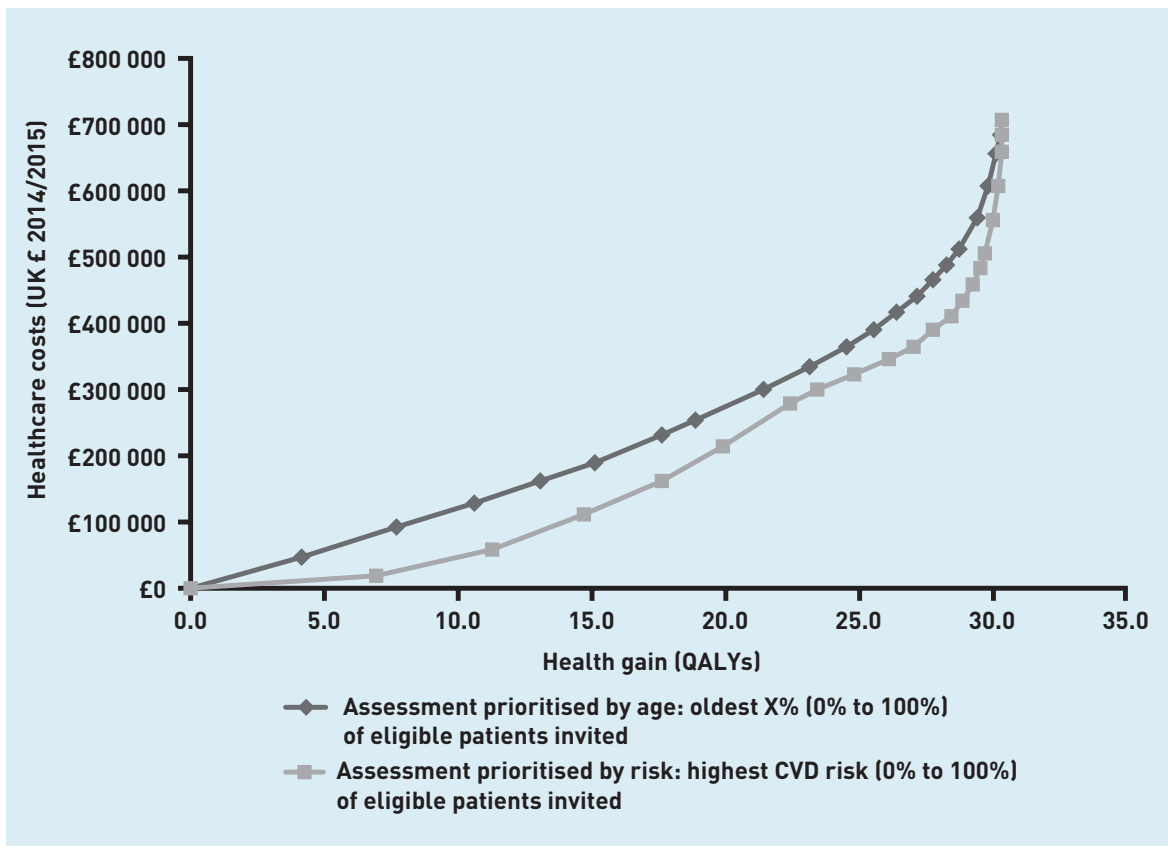

- timing of future changes in treatment;

- onset of diabetes;

- occurrence of fatal or non-fatal CVD events; or

- death from other causes.

QRISK2 and QDiabetes ${ }^{\circledR}$ were used to predict baseline risk of CVD and diabetes, and the REACH equation, which is a published prediction model developed from international data, was used to predict recurrent CVD events. ${ }^{26}$ Cessation of drugs led to cessation of treatment benefits. Random sampling determined the type of CVD event (non-fatal myocardial infarction, angina, coronary death, nonfatal stroke, transient ischaemic attack, or cerebrovascular death) based on their relative incidences and case fatality in large, UK-population cohort studies. (Further information is available from the authors on request.)

Costs and QALYS associated with each individual's simulated lifetime profile were estimated using published costs and health-related quality-of-life data. Liver dysfunction and myopathy were modelled as one-off events with a short-term impact on costs and quality of life. Diabetes was modelled as a lifetime event, with annual costs and utility decrement obtained from published sources. (Further information is available from the authors on request.)

\section{Analysis}

The impact of uncertainty was examined with a combination of non-parametric bootstrapping and a probabilistic sensitivity analysis (PSA) using distributions based on empirical data sources whenever possible, in line with models highlighted in previous studies. ${ }^{27,28}$ (Further information is available from the authors on request.) The model was run 10000 times for the base case analysis. For each iteration, results were calculated for a sample of 10000 patients Idrawn randomly with replacement from the THIN dataset) and using one complete set of input parameters (drawn by Monte Carlo sampling).

Results are presented as an expected incremental net benefit, averaged across the bootstrap/PSA iterations, with each QALY gained valued at the more conservative NICE threshold of $€ 20000$. A positive incremental net benefit indicates that the strategy is cost effective in comparison with opportunistic patient identification - the strategy with the largest incremental net benefit is the optimum strategy. Estimates of uncertainty around the optimum strategy are provided by the proportion of model iterations in which the strategy yielded the maximum incremental net benefit. This means that a high proportion of model iterations in which a particular strategy yielded the maximum would indicate a high degree of certainty that these results would be correct in any population of 10000 .

A series of sensitivity analyses tested the impact of changing key model parameters. Results were estimated for each sensitivity analysis over 1000 bootstrap/PSA iterations by holding one parameter fixed at a defined value, while allowing other parameters to vary, as in the base case analysis. 
Table 2. Estimated cost and QALY gain of a case-finding strategy based on prior estimated CVD risk versus opportunistic assessment ${ }^{\mathrm{a}}$

\begin{tabular}{|c|c|c|c|c|c|c|}
\hline Invited, \% & $\begin{array}{c}\text { Cut-off } \\
\text { 10-year QRisk } \\
\text { threshold above which } \\
\text { patients are invited }\end{array}$ & Extra cost, $€$ & QALY gain & $\begin{array}{c}\text { Incremental } \\
\text { cost-effectiveness } \\
\text { ratio, } €\end{array}$ & Net benefit, $\mathrm{E}^{\mathrm{b}}$ & $\begin{array}{c}\text { Probability } \\
\text { highest } \\
\text { net benefit, \% }\end{array}$ \\
\hline 0 & 0 & 0 & 0.00 & 0 & 0 & 3.9 \\
\hline 2 & 0.2027 & 18553 & 6.96 & 2666 & 120624 & 11.2 \\
\hline 4 & 0.1667 & 59689 & 11.26 & 9567 & 165497 & 12.0 \\
\hline 6 & 0.1445 & 109248 & 14.68 & 14491 & 184360 & 10.2 \\
\hline 8 & 0.1277 & 162280 & 17.53 & 18608 & 188397 & 8.3 \\
\hline 10 & 0.1142 & 215722 & 19.89 & 22645 & 181994 & 6.7 \\
\hline 13 & 0.0983 & 278487 & 22.39 & 25106 & 169308 & 4.7 \\
\hline 15 & 0.0896 & 298686 & 23.32 & 21719 & 167641 & 4.6 \\
\hline 20 & 0.0721 & 323632 & 24.87 & 16094 & 173820 & 7.3 \\
\hline 25 & 0.0590 & 343949 & 26.08 & 16791 & 177566 & 7.6 \\
\hline 30 & 0.0488 & 364684 & 26.99 & 22786 & 175200 & 6.9 \\
\hline 35 & 0.0406 & 386548 & 27.76 & 28395 & 168666 & 6.0 \\
\hline 40 & 0.0339 & 409016 & 28.38 & 36239 & 158551 & 4.2 \\
\hline 45 & 0.0283 & 431764 & 28.86 & 47392 & 145452 & 3.2 \\
\hline 50 & 0.0236 & 455915 & 29.23 & 65273 & 128648 & 1.6 \\
\hline 55 & 0.0197 & 480422 & 29.51 & 87525 & 107797 & 1.0 \\
\hline 60 & 0.0163 & 505461 & 29.74 & 108865 & 89378 & 0.4 \\
\hline 70 & 0.0110 & 555955 & 30.04 & 168313 & 44747 & 0.2 \\
\hline 80 & 0.0071 & 606743 & 30.21 & 298753 & -2536 & 0.01 \\
\hline 90 & 0.0041 & 656963 & 30.30 & 558000 & -51029 & 0.00 \\
\hline 95 & 0.0028 & 681608 & 30.31 & 2464500 & -75370 & 0.00 \\
\hline 100 & 0.0006 & 705732 & 30.32 & 2412400 & -99430 & 0.00 \\
\hline
\end{tabular}

aSelecting 0-100\% of the population for assessment compared with usual practice for 10000 patients. Mean per 10000 persons. ${ }^{b}$ Based on E20 000 per QALY gained. $C V D=$ cardiovascular disease. $Q A L Y=$ quality-adjusted life year.

\section{RESULTS}

Table 1 details the sample characteristics. Figure 2 shows mean cost and QALY gains per 10000 persons for a range of active case-finding strategies, prioritising by age and by prior risk, compared with opportunistic identification; both methods of case finding at all levels of implementation provided more QALYS at a higher cost than opportunistic identification. Compared with opportunistic assessment, using either strategy if all patients are invited gives a total health gain of 30.32 QALYS at a resource cost of $€ 705732$. Inviting patients for assessment according to estimated prior CVD risk dominated the age-based strategy providing more QALYs at lower cost if any proportion of the population between $0 \%$ and $100 \%$ was invited for assessment.

Table 2 presents the expected costs, QALYS, and incremental net benefit for active case finding using prior CVD risk, compared with opportunistic assessment.
At a cost of $€ 20000$ per QALY, the optimum strategy is to invite the $8 \%$ of patients with the highest prior risk (10-year CVD risk of $\geq 12.76 \%$ ). Compared with no case-finding, this yields an additional 17.53 QALYS at an additional cost of $€ 162280$ per 10000 people: $58 \%$ of the achievable benefit at $23 \%$ of the cost. Inviting a further $2 \%$ of the population by extending the invitation to those whose 10 -year CVD risk is $\geq 11.42 \%$ yields an incremental 2.36 QALYs at $€ 53442$ (£22 645 per QALY). Probabilistic sensitivity analysis reveals considerable uncertainty around these results but there is an $89.4 \%$ probability that the optimum strategy is to invite no more than the $35 \%$ of patients whose prior estimate of 10-year CVD risk is $\geq 4.06 \%$. This has a total resource cost of €386 548 and yields 27.76 QALYS (92\% of the QALY gain at $55 \%$ of the cost) (Table 2).

\section{Sensitivity analyses}

The sensitivity of results to the values of 


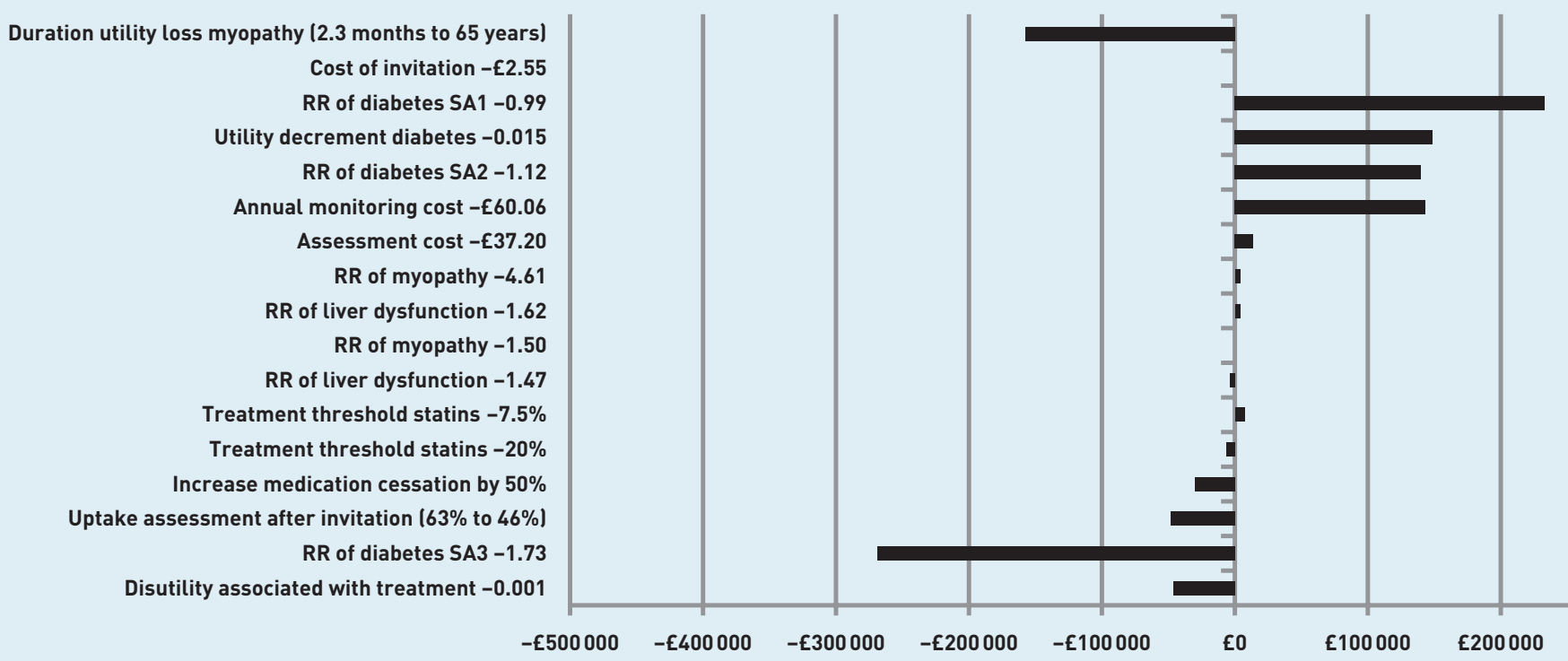

Figure 3. Difference in net benefit per sensitivity analysis compared with base case.

$R R=$ relative risk. $S A 1=$ sensitivity analysis $1 . S A 2=$ sensitivity analysis 2.SA3 = sensitivity analysis 3. key inputs is illustrated in Figure 3 and Table 3. Findings were robust to changes regarding assumptions about the effects of statins on liver dysfunction and myopathy due to the relative rarity of these events and their health effects of limited duration after treatment cessation. Findings were sensitive to assumptions about the effect of statins on diabetes incidence. When the relative risk of diabetes with statins was 1.73 lupper $\mathrm{Cl}$ from the meta-analysis of observational studies), ${ }^{23}$ the optimum strategy was to invite $2 \%$ of the population for assessment lprior 10-year CVD risk of $\geq 20.29 \%$ ); when it was reduced to 1.12 (as estimated in the NICE guideline based on data from randomised controlled trials) ${ }^{13}$ or 0.99 the lower $\mathrm{Cl}$ from the meta-analysis of observational studies), ${ }^{23}$ the optimum strategy was to invite $30 \%$ of the population for assessment (prior 10-year CVD risk of $\geq 4.88 \%$ ) and the net benefit of active case finding was much greater. Changing the utility decrement associated with diabetes from 0.131 to 0.015 also meant the optimum strategy was to invite $30 \%$ of the population..$^{29}$

Results were sensitive to the costs of risk

\section{Table 3. Sensitivity analysis, individuals prioritised for invitation based on prior CVD risk estimates} compared with usual care ${ }^{a}$

\begin{tabular}{|c|c|c|c|c|c|c|}
\hline $\begin{array}{l}\text { Sensitivity } \\
\text { analysis }\end{array}$ & Change & $\begin{array}{l}\text { Optimum } \\
\text { invite, \% }\end{array}$ & $\begin{array}{c}\text { Risk } \\
\text { threshold }\end{array}$ & Cost, $€$ & $\begin{array}{c}\text { Incremental } \\
\text { QALYs }\end{array}$ & Net benefit, $\mathrm{f}^{c}$ \\
\hline Base case & Not applicable & 8 & 0.1276 & 162280 & 17.53 & 188397 \\
\hline Uptake assessment & Reduce from $63 \%$ to $46 \%$ uptake & 8 & 0.1276 & 120310 & 12.99 & 139516 \\
\hline Start medication & Reduce by $50 \%^{d}$ & 6 & 0.1445 & 69060 & 7.36 & 78155 \\
\hline Stop medication & Increase by $50 \%{ }^{\mathrm{e}}$ & 20 & 0.0721 & 191759 & 18.47 & 177579 \\
\hline Assessment cost & Reduce by $50 \%$ (€37.20) & 40 & 0.0339 & 326023 & 28.50 & 243943 \\
\hline Annual monitoring cost & Reduce by $50 \%$ (€60.06) & 30 & 0.0488 & 116314 & 27.20 & 427743 \\
\hline RR of diabetes SA1 & Change from 1.31 to 0.99 & 30 & 0.0488 & 264881 & 43.41 & 603313 \\
\hline RR of diabetes SA2 & Change from 1.31 to 1.12 & 30 & 0.0488 & 303986 & 36.86 & 433247 \\
\hline RR of diabetes SA3 & Change from 1.31 to 1.73 & 2 & 0.2029 & 31591 & 4.57 & 59793 \\
\hline Utility decrement diabetes & Change from 0.131 to 0.015 & 30 & 0.0488 & 362956 & 40.16 & 440154 \\
\hline Disutility of treatment & Change from 0 to 0.001 & 6 & 0.1445 & 108849 & 13.01 & 151389 \\
\hline
\end{tabular}

${ }^{a}$ Results per 10000 persons. 'Strategy with the largest incremental net benefit is the 'optimum' at this threshold, yielding the greatest QALY gain while accounting for the opportunity cost of scarce healthcare resources. 'B Based on 120000 per QALY gained. IInitiate treatment with statins, reduce from 0.683 to 0.3415 , and with antihypertensives, reduce from 0.565 to 0.2825 (invitation-based strategies). ' Stay on statin medication, 1 year: 0.8614 to $0.4307,5$ years: 0.6877 to 0.343875 ; stay on antihypertensive medication, 1 year: 0.7055 to $0.35275,5$ years: 0.4905 to $0.24525 . C V D=$ cardiovascular disease. $Q A L Y=$ quality-adjusted life year. $R R=$ relative risk. $S A 1=$ sensitivity analysis 1 , SA2 = sensitivity analysis 2. SA3 = sensitivity analysis 3. 
Table 4. Sensitivity analyses: net benefit per 10000 persons for inviting for assessment population with 10 -year CVD risk thresholds by effect on diabetes risk and varying statin treatment thresholds ${ }^{a}$

\begin{tabular}{|c|c|c|c|c|c|c|}
\hline \multirow[b]{2}{*}{ Invited, \% } & \multirow[b]{2}{*}{ Cut-off } & \multicolumn{5}{|c|}{ 10-year CVD risk treatment threshold for statins (statins assumed to increase diabetes risk) } \\
\hline & & $\begin{array}{l}\text { Net benefit for } \\
10 \% \text { threshold, } €\end{array}$ & $\begin{array}{l}\text { Net benefit for } \\
15 \% \text { threshold, } €\end{array}$ & $\begin{array}{l}\text { Net benefit for } \\
20 \% \text { threshold, } \mathrm{E}\end{array}$ & $\begin{array}{l}\text { Net benefit for } \\
25 \% \text { threshold, } €\end{array}$ & $\begin{array}{l}\text { Net benefit for } \\
7.5 \% \text { threshold, } \mathrm{E}\end{array}$ \\
\hline 8 & 0.1276 & 195319 & 188397 & 112700 & 184396 & 167790 \\
\hline 10 & 0.1142 & 188736 & 183987 & 128576 & 196675 & 179743 \\
\hline 13 & 0.0983 & 159806 & 172395 & 144537 & 208960 & 193451 \\
\hline 15 & 0.0896 & 128521 & 171427 & 151326 & 218406 & 199252 \\
\hline 20 & 0.0721 & 66060 & 178734 & 169385 & 232759 & 212812 \\
\hline 25 & 0.0590 & 48489 & 181858 & 176995 & 238400 & 215999 \\
\hline 30 & 0.0488 & 42467 & 181101 & 180853 & 237444 & 215407 \\
\hline 35 & 0.0406 & 36219 & 174050 & 178669 & 231126 & 210097 \\
\hline 40 & 0.0339 & 26217 & 162976 & 172179 & 221274 & 200799 \\
\hline 45 & 0.0283 & 12992 & 150559 & 165075 & 207209 & 187863 \\
\hline \multirow[t]{2}{*}{50} & 0.0236 & -3225 & 132937 & 156436 & 191749 & 172612 \\
\hline & Cut-off $^{b}$ & \multicolumn{4}{|c|}{ 10-year CVD risk treatment threshold for statins (statins assumed not to affect diabetes risk) } & \\
\hline 8 & 0.1276 & 420494 & 414083 & 347839 & 245586 & 193065 \\
\hline 10 & 0.1142 & 478593 & 468227 & 365636 & 259219 & 205668 \\
\hline 13 & 0.0983 & 550404 & 527239 & 382343 & 273194 & 220312 \\
\hline 15 & 0.0896 & 585960 & 547727 & 392544 & 283562 & 226582 \\
\hline 20 & 0.0721 & 647281 & 574502 & 408967 & 299397 & 240952 \\
\hline 25 & 0.0590 & 668209 & 586376 & 414148 & 306451 & 245221 \\
\hline 30 & 0.0488 & 675641 & 590219 & 412947 & 306481 & 245441 \\
\hline 35 & 0.0406 & 675835 & 586021 & 406288 & 300916 & 241082 \\
\hline 40 & 0.0339 & 670013 & 577154 & 397083 & 291742 & 232443 \\
\hline 45 & 0.0283 & 659702 & 566787 & 384913 & 278268 & 219889 \\
\hline 50 & 0.0236 & 645655 & 550856 & 368788 & 263606 & 205184 \\
\hline
\end{tabular}

assessment and of monitoring those on medication (Figure 3): halving these costs made the optimum strategy to invite $40 \%$ and $30 \%$, respectively, of the population (prior 10-year CVD risk thresholds of $\geq 3.39 \%$ and $4.9 \%$ respectively) (Table 3 ). Reducing the uptake of invitations for assessment from $63 \%$ to $46 \%$, (the national average uptake of NHS Health Checks) did not affect the optimum strategy but decreased the benefits of case finding. ${ }^{30}$

Halving the proportion of eligible patients started on medication after assessment also reduced cost effectiveness, with the optimum strategy becoming to invite only $6 \%$ of the population for assessment (prior 10 -year CVD risk of $>14.45 \%$ ). Doubling the number who stop their medication makes the optimum strategy to invite 20\% of the population (prior 10-year CVD risk of 27.21\%) (Table 3).

Table 4 presents the net benefit at different risk assessment and statin initiation thresholds. The optimum strategy is sensitive to assumptions about the effect of statins on diabetes incidence. If the relative risk of diabetes with statins is 1.31, the optimum strategy is to invite $25 \%$ of the population with the highest prior estimate of CVD risk for assessment (prior 10-year CVD risk of $\geq 5.90 \%$ ), but to only offer statin treatment to those whose assessed 10-year CVD risk is $\geq 20 \%$.

If statins have no effect on diabetes, the optimum strategy is to invite the highest priority $35 \%$ of the population for assessment lprior 10-year CVD risk is $\geq 4.06 \%$ ) and to offer statins to those whose assessed 10 -year CVD risk is $\geq 7.5 \%$.

\section{DISCUSSION \\ Summary}

It was found that it was cost effective to undertake targeted case finding for CVD prevention in a small proportion of the population, prioritised by their estimated 
CVD risk. This strategy dominated (that is, it was clearly superior because it resulted in more QALY gain at lower cost) targeting patients by age alone. Under the base case assumptions, the optimum strategy was to invite those whose prior estimate of 10-year CVD risk was $\geq 12.76 \%$ ( $8 \%$ of the population aged $30-74$ years). This estimate was sensitive to a number of assumptions but, for most, $>90 \%$ of the potential health gain was in the $35 \%$ of the population at highest risk; it was not cost effective to invite those whose estimated 10-year CVD risk was $<4.06 \%$. It is more cost effective to prioritise patients for CVD risk factor assessment than to offer it to all individuals over a certain age; the most efficient prioritisation makes use of multiple risk factors but the incremental benefits of case finding across the entire population are small in relation to the incremental costs.

The UK's Health Check programme begins at age 40 years but the model presented here includes patients aged between 30 and 39 years. In practice, including people aged $<40$ years makes little difference to the patients invited for assessment. This can be illustrated by determining the number of patients who would be invited under the optimum strategy who are aged $<40$ years. A post hoc analysis of the primary care dataset (THIN) used in our analysis identified records on 1067654 untreated patients without diabetes, aged 30-74 years, of whom 89552 had a 10-year CVD risk of $\geq 12.76 \%$; 14 of these $(0.016 \%)$ were aged $<40$ years. Of 300757 untreated persons without diabetes aged $<40$ years, $99.72 \%$ had a 10 -year CVD risk of $<5 \%$ and would not be invited for assessment under almost any of the scenarios.

\section{Strengths and limitations}

The model of CVD prevention for primary care is the first to consider the incremental cost effectiveness of targeting case finding at anything from one patient to the entire population; this was a key omission of previous modelling. ${ }^{11}$ In addition, the whole process - identification, invitation, assessment, initiation, and continuation of treatment - was assessed. Healthcare costs associated with each step, health benefits, and adverse effects of treatment were included, and the authors made realistic estimates of the completeness of prior risk factor data, rates of nonattendance, the effect of within-individual risk factor variation on assessment, noninitiation, and discontinuation of treatment.

All models make simplifying assumptions. Rather than considering every possible beneficial effect of treatment, the focus has been the most important CVD outcomes that dominate the clinical decision. Drug treatments formed a focus because much advice on health behaviours, such as smoking, can be delivered opportunistically without case finding; in addition, evidence for the effectiveness of behavioural change interventions is not strong. ${ }^{31}$ Smokers and most of those with a BMl of $\geq 30 \mathrm{~kg} / \mathrm{m}^{2}$ can be identified from electronic primary care records without assessment. In the UK, systematic case finding resulted in an additional $0.5 \%$ of attendees being referred to smoking cessation, $5.5 \%$ to weight-loss services, and $5.9 \%$ to advice on exercise. ${ }^{32}$ The effects of referral to smoking cessation, weight loss, or exercise services were not modelled, and therefore may not have captured the full benefits of case finding. This is a potential weakness. However, it is uncertain how many of those referred to these services attended, or the effectiveness of attendance, therefore the effects of these omissions are likely to be small.

The effects of statins on diabetes and the effects of diabetes on quality of life are important factors that affect our findings about the optimum proportion of the population that should be invited for assessment. The effects of statins on diabetes risk may vary between different genotypes. ${ }^{33}$ Increased diabetes risk attenuates the benefits of statins, which may be particularly important in older patients and those who receive more intensive treatment. ${ }^{34,35}$ The effects of a potential interaction between age, intensity of statin treatment, and diabetes risk were not explored in this analysis but could be formally modelled. Nevertheless, even without formal modelling it is intuitive that, if statins have a large effect on diabetes risk, the optimum strategy is to raise the CVD risk threshold at which statins should be offered; if they have no effect, the optimum CVD risk threshold for offering statins should be lower.

The optimum proportion of the population to be invited for CVD risk factor assessment will vary internationally, due to differences in CVD epidemiology, risk assessment and prescribing practice, healthcare systems, and costs. Nevertheless, the researchers anticipate that this study's key findings are robust and generalisable to most high- and middle-income countries that have a listbased primary healthcare system.

\section{Comparison with existing literature}

The findings of the study presented here concur with previous modelling studies that 


\section{Funding}

No specific funding was received to conduct this study. Tom Marshall was funded by the National Institute for Health Research (NIHR) through the Collaboration for Leadership in Applied Health Research and Care for West Midlands (CLAHRCWM) programme. These funders had no role in study design, data collection and analysis, decision to publish, or preparation of the manuscript. The views expressed in this publication are not necessarily those of the NIHR, the Department of Health, University of Birmingham, or the CLAHRC Birmingham and Black Country.

\section{Ethical approval}

The modelling in this study mainly made use of publicly available research and data sources, and therefore ethical approval was not required. All research involving The Health Improvement Network (THIN) was approved by the NHS South-East Multicentre Research Ethics Committee (MREC) in 2003 subject to independent scientific review. Scientific review committee approved the use of cardiovascular risk factor data from THIN (reference 16THIN009).

\section{Provenance}

Freely submitted; externally peer reviewed.

\section{Competing interests}

The authors have declared no competing interests.

\section{Acknowledgements}

The authors thank Nicola Adderley for undertaking additional analysis in response to reviewer's comments.

\section{Discuss this article}

Contribute and read comments about this article: bjgp.org/letters support targeted case finding. ${ }^{36-38}$ Targeted case finding was also a recommendation of the 2008 NICE guidelines on lipid lowering. ${ }^{39}$ The findings presented here are in line with an evaluation of CVD case-finding programmes in six European countries (Denmark, France, Germany, Italy, Poland, and the UK), which also favoured targeting individuals at higher risk. ${ }^{40}$ However, this study revealed that CVD risk-based targeting dominates (results in more QALY gain at lower resource cost) age-based targeting strategies that have been recommended by some authors. ${ }^{41,42}$ Therefore, although other analyses promote targeting by age or age and sex, we go further by demonstrating the advantages of targeting based on multivariable predicted cardiovascular risk.

Just as including additional risk factors over and above age makes targeted case finding more precise and more cost effective, having more recorded risk factor information available will increase precision and cost effectiveness. Targeting case finding using deprivation quintile of residence has been modelled as a policy option. ${ }^{43}$ In effect, this uses a single categorical risk factor to identify those at highest risk while ignoring the strongest single risk factor (age) and not taking account of the optimum predictor (cardiovascular risk). Strategies using single categorical risk factors are almost certain to be less efficient than those using cardiovascular risk.

\section{Implications for research and practice}

Primary care should rank patients by a prior estimate of their CVD risk and target for case finding those who are at highest risk. Assessing all adults is a poor use of resources because those whose prior CVD estimate is low are very unlikely to be eligible for treatment. Uncertainties about the effects of statins on incidence of diabetes merit further research. 


\section{REFERENCES}

1. Perk J, De Backer G, Gohlke H, et al. European guidelines on cardiovascular disease prevention in clinical practice (version 2012). The Fifth Joint Task Force of the European Society of Cardiology and Other Societies on Cardiovascular Disease Prevention in Clinical Practice. Eur Heart J 2012; 33(13): 1635-1701.

2. Department of Health. Putting prevention first - vascular checks: risk assessment and management - impact assessment. http://www.healthcheck. nhs.uk/document.php?o=228 (accessed 12 Oct 2016).

3. Allan GM, Lindblad AJ, Comeau A, et al. Simplified lipid guidelines: prevention and management of cardiovascular disease in primary care. Can Fam Physician 2015; 61(10): 857-867

4. Royal Australian College of General Practitioners. Guidelines for preventive activities in general practice. 9th edn. http://www.racgp.org.au/your-practice/ guidelines/redbook/prevention-of-vascular-and-metabolic-disease/ assessment-of-absolute-cardiovascular-risk/ laccessed 12 Oct 2016).

5. Ministry of Health Malaysia. National strategic plan for non communicable disease. Putrajaya: Ministry of Health Malaysia, 2010.

6. Yamout R, Adib SM, Hamadeh R, et al. Screening for cardiovascular risk in asymptomatic users of the primary health care network in Lebanon, 20122013. Prev Chronic Dis 2014; 11: E120.

7. Alowaish R, Al Asi T, Adly L. Risk factors for cardiovascular diseases: Kuwait Heart Foundation's mobile screening. J Saudi Heart Assoc 2012; 24(4): 295-296.

8. Jørgensen $T$, Jacobsen RK, Toft U, et al. Effect of screening and lifestyle counselling on incidence of ischaemic heart disease in general population: Inter99 randomised trial. BMJ 2014; 348: g3617.

9. Krogsbøll LT, Jørgensen KJ, Grønhøj Larsen C, Gøtzsche PC. General health checks in adults for reducing morbidity and mortality from disease: Cochrane systematic review and meta-analysis. BMJ 2012; 345: e7191.

10. Chang KC, Lee JT, Vamos EP, et al. Impact of the National Health Service Health Check on cardiovascular disease risk: a difference-in-differences matching analysis. CMAJ 2016; 180(10): E228-E238.

11. Department of Health. Economic modelling for vascular checks. http://unw. healthcheck.nhs.uk/document.php?o=225 [accessed 12 Oct 2016].

12. National Institute for Health and Care Excellence. Guide to the methods of technology appraisal 2013. https://www.nice.org.uk/process/pmg9/chapter/ foreword laccessed 12 Oct 2016)

13. National Institute for Health and Care Excellence. Cardiovascular disease: risk assessment and reduction, including lipid modification. CG181. London: NICE, 2014 [updated: September 2016]. https://www.nice.org.uk/Guidance/CG181 laccessed 12 Oct 2016).

14. Hippisley-Cox J, Coupland C, Robson J, et al. Predicting risk of type 2 diabetes in England and Wales: prospective derivation and validation of QDScore. BMJ 2009; 338: b880

15. Marshall T, Westerby P, Chen J, et al. The Sandwell Project: a controlled evaluation of a programme of targeted screening for prevention of cardiovascular disease in primary care. BMC Public Health 2008; 8(1): 73.

16. National Clinical Guideline Centre. Hypertension: the clinical management of primary hypertension in adults. Clinical Guideline 127: methods, evidence, and recommendations. London: NCGC, 2011. https://www.nice.org.uk/guidance/ cg127/evidence/full-guideline-248588317 [accessed 12 Oct 2016]

17. Stone NJ, Robinson JG, Lichtenstein AH, et al. 2013 ACC/AHA guideline on the treatment of blood cholesterol to reduce atherosclerotic cardiovascular risk in adults: a report of the American College of Cardiology/American Heart Association Task Force on Practice Guidelines. J Am Coll Cardiol 2014 63(25-PA): 2889-2934

18. Cholesterol Treatment Trialists' (CTT) Collaborators. The effects of lowering LDL cholesterol with statin therapy in people at low risk of vascular disease: meta-analysis of individual data from 27 randomised trials. Lancet 2012; 380(9841): $581-590$

19. Law M, Morris J, Wald N. Use of blood pressure lowering drugs in the prevention of cardiovascular disease: meta-analysis of 147 randomised trials in the context of expectations from prospective epidemiological studies. BMJ 2009; 338: b1665.

20. Law M, Wald N, Morris J. Lowering blood pressure to prevent myocardial infarction and stroke: a new preventive strategy. Health Technol Assess 2003: 7(31): 1-94.

21. Lewington S, Clarke R, Qizilbash N, et al. Age-specific relevance of usual blood pressure to vascular mortality: a meta-analysis of individual data for one million adults in 61 prospective studies. Lancet 2002; 360(9349): 1903-1913.

22. Hippisley-Cox J, Coupland C. Unintended effects of statins in men and women in England and Wales: population based cohort study using the QResearch database. BMJ 2010; 340: c2197.

23. Macedo AF, Taylor FC, Casas JP, et al. Unintended effects of statins from observational studies in the general population: systematic review and metaanalysis. BMC Med 2014; 12: 51

24. Wright JM, Musini VM. First-line drugs for hypertension. Cochrane Database Syst Rev 2009; 3: CD001841.

25. Law M, Wald NJ, Morris JK, Jordan RE. Value of low dose combination treatment with blood pressure lowering drugs: analysis of 354 randomised trials. BMJ 2003; 326(7404): 1427.

26. Wilson PW, D'Agostino Sr R, Bhatt DL, et al. An international model to predict recurrent cardiovascular disease. Am J Med 2012; 125(7): 695-703.

27. Lord J, Asante MA. Estimating uncertainty ranges for costs by the bootstrap procedure combined with probabilistic sensitivity analysis. Health Econ 1999; 8(4): $323-333$

28. Parmigiani G, Samsa GP, Ancukiewicz M, et al. Assessing uncertainty in costeffectiveness analyses application to a complex decision model. Med Decis Making 1997; 17(4): 390-401.

29. Grandy S, Fox KM, SHIELD Study Group. Change in health status (EQ-5D) over 5 years among individuals with and without type 2 diabetes mellitus in the SHIELD longitudinal study. Health Qual Life Outcomes 2012; 10: 99.

30. National Health Service. NHS Health Check. Explore NHS Health Check data. http://wnw.healthcheck.nhs.uk/commissioners_and_providers/data/ laccessed 12 Oct 2016).

31. Ebrahim S, Taylor F, Ward K, et al. Multiple risk factor interventions for primary prevention of coronary heart disease. Cochrane Database Syst Rev 2011; 1: CD001561.

32. Robson J, Dostal I, Sheikh A, et al. The NHS Health Check in England: an evaluation of the first 4 years. BMJ Open 2016; 6(1): e008840.

33. Swerdlow DI, Preiss D, Kuchenbaecker KB, et al. HMG-coenzyme A reductase inhibition, type 2 diabetes, and bodyweight: evidence from genetic analysis and randomised trials. Lancet 2015; 385(9965): 351-361.

34. Sattar N, Preiss D, Murray HM, et al. Statins and risk of incident diabetes: a collaborative meta-analysis of randomised statin trials. Lancet 2010; 375(9716) 735-742.

35. Preiss D, Seshasai SR, Welsh $\mathrm{P}$, et al. Risk of incident diabetes with intensivedose compared with moderate-dose statin therapy: a meta-analysis. JAMA 2011; 305(24): 2556-2564.

36. Marshall T, Rouse A. Resource implications and health benefits of primary prevention strategies for cardiovascular disease in people aged 30 to 74 : mathematical modelling study. BMJ 2002; 325(7357): 197.

37. Chamnan $\mathrm{P}$, Simmons RK, Hori $\mathrm{H}$, et al. A simple risk score using routine data for predicting cardiovascular disease in primary care. Br J Gen Pract 2010; DOI: 10.3399/bjgp10X515098.

38. Lawson KD, Fenwick EA, Pell AC, Pell JP. Comparison of mass and targeted screening strategies for cardiovascular risk: simulation of the effectiveness, cost-effectiveness and coverage using a cross-sectional survey of 3921 people. Heart 2010; 96(3): 208-212.

39. National Institute for Health and Clinical Excellence. Lipid modification: cardiovascular risk assessment and the modification of blood lipids for the primary and secondary prevention of cardiovascular disease. CG67. London: NICE, 2008 [reissued 2010]. http://www.nescn.nhs.uk/wp-content/ uploads/2014/05/Lipid-Modification-Guidance.pdf laccessed 12 Oct 2016).

40. Schuetz CA, Alperin P, Guda S, et al. A standardized vascular disease health check in Europe: a cost-effectiveness analysis. PloS One 2013; 8(7): e66454.

41. Wald NJ, Simmonds M, Morris JK. Screening for future cardiovascular disease using age alone compared with multiple risk factors and age. PLoS One 2011; 6(5): e18742.

42. Selvarajah S, Haniff J, Kaur G, et al. Identification of effective screening strategies for cardiovascular disease prevention in a developing country: using cardiovascular risk-estimation and risk-reduction tools for policy recommendations. BMC Cardiovasc Disord 2013; 13: 10.

43. Kypridemos C, Allen K, Hickey GL, et al. Cardiovascular screening to reduce the burden from cardiovascular disease: microsimulation study to quantify policy options. BMJ 2016; 353: i2793. 